\author{
PA Insel ${ }^{1,2}$, A Snead ${ }^{1}$, F Murray ${ }^{2}$, L Zhang ${ }^{1}$, H Yokouchi ${ }^{1}$, T Katakia ${ }^{1}$, \\ O Kwon ${ }^{1}$, D Dimucci ${ }^{1}$ and A Wilderman ${ }^{1}$ \\ ${ }^{1}$ Departments of Pharmacology and ${ }^{2}$ Medicine, University of California, San Diego, La Jolla, CA, \\ USA
}

\section{Correspondence}

PA Insel, Departments of Pharmacology \& Medicine, Mail Code 0636, University of California, San Diego, 9500 Gilman Dr La Jolla, CA 92093. USA. E-mail: pinsel@ucsd.edu

\section{Keywords}

G-protein-coupled receptors; adenylyl cyclase; cyclic AMP;

7-transmembrane receptors

\section{Received}

30 January 2007

Revised

7 March 2011

Accepted

4 April 2011

G-protein-coupled receptors [GPCRs, also known as 7-transmembrane (7-TM) receptors] comprise the largest family of membrane receptors in humans and other species and, in addition, represent the greatest number of current drug targets. In this article, we review methods to define GPCR expression and data indicating that individual cells express $>100$ different GPCRs. Results from studies that have quantified expression of these receptors lead us to conclude that the optimal GPCRs may not be currently used as therapeutic targets. We propose that studies of GPCR expression in individual cells will likely reveal new insights regarding cellular physiology and therapeutic approaches. Findings that define and characterize the most highly expressed GPCRs thus have important potential in terms of identifying new drug targets and novel therapies directed at a wide range of clinical disorders.

\title{
LINKED ARTICLES
}

This article is part of a themed section on the Molecular Pharmacology of G Protein-Coupled Receptors (GPCRs). To view the other articles in this section visit http://dx.doi.org/10.1111/bph.2012.165.issue-6. To view the 2010 themed section on the same topic visit http://onlinelibrary.wiley.com/doi/10.1111/bph.2010.159.issue-5/issuetoc

\section{Abbreviations \\ 7-TM, 7-transmembrane; GPCR, G protein-coupled receptor}

\section{Introduction}

A fundamental goal of pharmacology is to advance the understanding of the regulation of cells and tissues, in particular by endogenous molecules (e.g. neurotransmitters and hormones), and to identify ways to target the expression and action of such molecules with drugs. Results of efforts to identify the genomes of humans and other species have revealed that G-protein-coupled receptors (GPCRs), plasma membrane-localized glycoprotein receptors that couple to heterotrimeric guanine nucleotide binding proteins (G proteins), are the largest family ('superfamily') of receptors that regulate cells. GPCRs are sometimes identified by alternative names, including 7-transmembrane (7-TM) or heptahelical receptors, based on the general structure and membrane topology of the receptors and on the ability of such receptors to regulate cellular events in a G-protein-independent manner (e.g. Rajagopal et al., 2010).
The number of GPCRs varies widely among different species. Phylogenetic studies demonstrate a large diversity in expression throughout the animal kingdom, and that the genomes of species such as Caenorhabditis elegans, mice and rats express more GPCRs than do humans (Table 1). In addition to being receptors for neurotransmitters and hormones, GPCRs are receptors for light and for chemicals that are sensory cues related to taste and smell. The increased number of receptors for such sensory modalities likely reflects the more important role for olfaction, in particular, in species other than humans. Of note, GPCRs are expressed on every human chromosome (Fredriksson et al., 2003).

The precise number of GPCRs expressed in most species, including humans, is not known with certainty. Vassilatis et al. (2003) proposed that there are 367 human GPCRs for endogenous ligands ('endoGPCRs'), that is nonchemosensory receptors, while Fredriksson et al. (2003) identified 342 non-olfactory human GPCRs and suggested that 


\section{Table 1}

Expression of GPCRs in genomes of different species (adapted from http://sevens.cbrc.jp and listed in descending order of number of GPCRs)

\begin{tabular}{|c|c|}
\hline Species & Number of GPCRs \\
\hline Elephant & 3470 \\
\hline Cow & 2606 \\
\hline Rat & 2334 \\
\hline Mouse & 2320 \\
\hline Squirrel & 2252 \\
\hline Tree shrew & 2195 \\
\hline Armadillo & 2191 \\
\hline Opossum & 2083 \\
\hline Wallaby & 2050 \\
\hline Guinea pig & 1466 \\
\hline Dog & 1438 \\
\hline Rabbit & 1367 \\
\hline Hedgehog & 1316 \\
\hline Nematode (Caenorhabditis elegans) & 1308 \\
\hline Human & 1265 \\
\hline Bat & 1243 \\
\hline Alpaca & 1222 \\
\hline Orangutan & 1211 \\
\hline Chimpanzee & 1203 \\
\hline Baboon & 1066 \\
\hline Zebrafish & 1050 \\
\hline Marmoset & 1010 \\
\hline Rhesus & 1000 \\
\hline Cat & 931 \\
\hline Gorilla & 867 \\
\hline Chicken & 683 \\
\hline Fugu & 655 \\
\hline Dolphin & 507 \\
\hline Fruit fly (Drosophila melanogaster) & 251 \\
\hline Mosquito (Anopheles gambiae) & 236 \\
\hline Slime mold (Dictyostelium discoideum) & 30 \\
\hline Mouse-ear Cress (Arabidopsis thaliana) & 19 \\
\hline Rice (Oryza sativa) & 15 \\
\hline Budding yeast (Saccharomyces cerevisiae) & 6 \\
\hline
\end{tabular}

they be classified into five families: glutamate, rhodopsin, adhesion, frizzled and secretin. A reason for the uncertainty regarding the exact number of GPCRs is that their identification is primarily based on similarity in the gene sequence, such that the topology of the inferred, encoded amino acids is predicted to generate a protein with 7-TM $\alpha$-helical $\sim 20$ hydrophobic amino acid-containing regions, an extracellular amino terminus, intracellular carboxy terminus, three intracellular and three extracellular regions that join the TM domains. A substantial number of the receptors that are encoded by such genes are 'orphans', that is without identified agonists. Some GPCRs have been de-orphanized, but the cognate agonists for $>25 \%$ of the GPCRs identified in humans are not as yet known (Chung et al., 2008). De-orphanization has identified unexpected GPCR agonists, including proteins, peptides, lipids, fatty acids and other types of molecules (e.g. Chung et al., 2008; Ahmed et al., 2009; Oh et al., 2010). Identification of agonists for orphan GPCRs should provide insights regarding cell and tissue regulation and new therapeutic opportunities.

In this review, we briefly address the following questions:

1) Which approaches are available to identify GPCRs that are expressed in tissues and cells and what are the results of studies that have sought to identify these receptors?

2) How can GPCR expression results be organized and used to further insights regarding GPCR action and drug development?

\section{Which approaches are available to identify GPCRs that are expressed in tissues and cells and what are the results of studies that have sought to identify these receptors?}

Historically, one could identify receptors in a given cell or tissue by assessing the binding of radioligands (i.e. radiolabelled agonists or antagonists), molecular cloning and expression studies, antisense approaches and/or by experiments with transgenic or knockout animals. More recently, a number of proprietary (i.e. private) methods have been used to identify GPCRs expressed in tissues; much of the data from such studies are not published. One published data set revealed that human tissues express many orphan and olfactory GPCRs with, for example, the human heart expressing $>100$ different GPCRs, of which approximately a third each had known agonists, were orphan or olfactory GPCRs (e.g. Hakak et al., 2003). The functional roles of olfactory GPCRs in tissues such as the heart and kidney are not well defined (Hakak et al., 2003; Pluznick et al., 2009); olfactory receptors may also be expressed and functional in cancer cells (Neuhaus et al., 2009). Recent evidence reveals that taste receptor GPCRs, initially identified on the tongue, are also expressed in other tissues, where they can have functional activity and thus, are potential therapeutic targets (Kidd et al., 2008; Shah et al., 2009; Wellendorph et al., 2009; Deshpande et al., 2010).

Some academic laboratories have used commercial or customized approaches to define GPCR expression (Akiyama et al., 2008; Regard et al., 2008; Moore-Morris et al., 2009). Commercial microarrays that assay genome-wide RNA expression are not optimized to detect GPCRs relative to other RNAs detected on such microarrays. In addition, independent validation of the results has generally not been provided.

Regard et al. (2008) have performed the most extensive analysis of GPCR expression, quantifying RNA transcripts for 353 non-odorant GPCRs in 41 tissues from adult mice. Data from this study is accessible at: http://pdsp.med.unc.edu/ ShaunCell/home.php. Based on their results, the authors predicted previously unidentified roles for less-studied receptors and inferred side effects of drugs active at GPCRs. 
Although efforts such as those of Regard et al. have defined GPCR expression in tissues, very limited data are available with respect to GPCR expression in individual cells. We believe that this is an important limitation of previous studies. Efforts to understand physiological responses in tissues should be aided by data that define the GPCR expression and properties of their individual cell types and how such cells interact to produce such responses. The application of those types of efforts can also advance understanding of disease processes and help identify the contribution of particular GPCRs in disease settings. Our recent work indicates that a commercial GPCR microarray can be used to assess RNA for non-olfactory receptors and can identify expression of GPCRs in individual cell types (Snead and Insel, 2010). We have conducted studies in a number of human, rat and murine cell types. We find that individual cell types, including cancer cells, typically express $>100$ different receptors, and that the most highly expressed GPCRs are generally not ones assessed in previous experimental efforts or that have been targeted therapeutically (Snead et al., unpubl. data). We believe that expression of GPCRs in cancer cells may be a particularly fruitful area for further study and drug development. The recognition that chemosensory GPCRs contribute to physiological regulation (e.g. Hakak et al., 2003; Kidd et al., 2008; Pluznick et al., 2009; Shah et al., 2009; Wellendorph et al., 2009; Deshpande et al., 2010) implies that experimental approaches should consider the contribution of such GPCRs since they may play a role in physiological regulation and also provide opportunities for the development of novel drugs.

\section{How can GPCR expression results be organized and used to further insights regarding GPCR action and drug development?}

By contrast with the vast majority of pharmacological experiments in which investigators assess the role of GPCRs known to be expressed in a particular tissue or cell type, the approaches described above, which seek to define receptor expression based on RNA expression, are 'unbiased'. An important caveat, though, is the need to show that GPCRs identified on the basis of RNA expression are also expressed at the protein level, and that they have functional activity. There are several challenges with such experiments, including:

1 Identification of receptor protein can be difficult as there are only limited specific, validated antibodies or other reagents to detect receptor protein. Such analyses require protein standards so that one can obtain quantitative information regarding expression of receptor protein.

2 Other methods to quantify receptor protein generally require the use of radiolabelled receptor-selective compounds in radioligand binding assays. It can be difficult to create such compounds and to develop and use such assays for particular receptors.

3 Receptor-selective drugs are needed to conduct studies that define G-protein linkage, signalling pathways and functional responses of the GPCRs. Such drugs should include agonists and antagonists in order to define pharmacological and biochemical properties of a receptor. The availabil- ity of such agents may be limited, in particular for orphan GPCRs. In addition, one may encounter difficulties related to distribution in cells and tissues and cross-reactivity of drugs with various types and sub-types of GPCRs. The use of mutagenesis to generate constitutively active receptors is an alternative approach to aid in the study of orphan receptors (e.g. Reiners et al., 2007).

4 Appropriate methods are needed to isolate and study the GPCRs in individual cells and in turn, in tissues and whole animals, especially if a goal is drug discovery and validation. GPCR expression may differ among individual types of cells in a tissue, such that only a small percentage of the cells express the bulk of the receptors; 'average' expression in a tissue may obscure such heterogeneity. Moreover, disease-related changes in GPCR expression may only occur in a sub-population of cells, and efforts to target such receptors may be complicated by their expression in other tissues and thus potentially by the production of adverse side effects (Malik, 2009). In addition, new types of assays may be required to assess GPCR-mediated signal transduction by heterotrimeric G- proteins and G-proteinindependent signalling (Rajagopal et al., 2010).

5 Development of effective pharmacological approaches may be possible with small molecules, but peptide or protein therapeutics, such as antibodies or nucleic acid therapeutics, may be required to assess newly identified GPCRs, for example orphan GPCRs. In addition to the technical challenges involved in developing and validating such novel therapeutics are concerns about their ultimate clinical utility, including their cost.

A useful way to organize the large amount of data that accrue from studies that seek to identify GPCRs expressed in a particular cell type or tissue is to classify the receptors based on their known G-protein linkages (e.g. Gs, Gi, Gq/11, G12/13 or multiple such linkages), those that show non-G protein linkages (e.g. frizzled and smoothened receptors) or that are orphan GPCRs. By classifying GPCRs in this way, one can 'rank order' individual receptors and groups of GPCRs and identify those that are mostly highly expressed (and thus may be most physiologically important) or perhaps most 'specific' for a particular cell type or disease setting. For example, pro-fibrotic agents promote the conversion of 'resting' fibroblasts to activated myofibroblasts, which may have differences in the repertoire or level of expression of GPCRs, based on changes in responses to GPCR agonists (Swaney et al., 2005; Yokoyama et al., 2008).

The 2011 BJP Guide to Receptors and Channels (Alexander et al., 2011) (accessible at http://onlinelibrary.wiley. com/doi/10.1111/bph.2011.164.issue-s1/issuetoc) and the IUPHAR Database of Receptors and Ion Channels (available at http://www.iuphar-db.org) (Harmar et al., 2009; Sharman et al., 2011) provide information that aids in classification and defining groups of GPCRs. Another site with limited such information is: http://www.discoverx.com/gpcrs/prod-gpcr table.php. Clustering of GPCRs that have particular G-protein linkages can help reveal preferential GPCR expression patterns. For example, cells in which cAMP promotes apoptosis (Insel et al., 2011) may express fewer or lower expression of Gs-linked receptors that increase cAMP than of Gi-linked receptors that decrease cAMP. 


\section{Conclusions}

The use of unbiased, hypothesis-generating approaches provides the opportunity to identify the full range of GPCRs that are expressed in native tissues and cells, including normal and pathological cells. If such GPCRs are selectively expressed in particular cells or tissues, are functional and can be activated or blocked, discovery of their expression may not only reveal new insights regarding cellular physiology but also identify new, potentially useful therapeutic targets.

\section{Acknowledgements}

Work described in this article was supported with funding provided by grants from the National Institutes of Health, the Lymphoma and Leukemia Society and the Ellison Medical Foundation.

\section{Conflicts of interest}

The authors indicated no potential conflicts of interests.

\section{References}

Ahmed K, Tunaru S, Offermanns S (2009). GPR109A, GPR109B and GPR81, a family of hydroxy-carboxylic acid receptors. Trends Pharmacol Sci 30: 557-562.

Akiyama K, Nakanishi S, Nakamura NH, Naito T (2008). Gene expression profiling of neuropeptides in mouse cerebellum, hippocampus, and retina. Nutrition 24: 918-923.

Alexander SPH, Mathie A, Peters JA (2011). Guide to Receptors and Channels (GRAC), 5th Edition. Br J Pharmacol 164 (Suppl. 1): S1-S324.

Chung S, Funakoshi T, Civelli O (2008). Orphan GPCR research. Br J Pharmacol 153 (Suppl. 1): S339-S346.

Deshpande DA, Wang WC, McIlmoyle EL, Robinett KS, Schillinger RM, An SS et al. (2010). Bitter taste receptors on airway smooth muscle bronchodilate by localized calcium signaling and reverse obstruction. Nat Med 16: 1299-1304.

Fredriksson R, Lagerstrom MC, Lundin LG, Schioth HB (2003). The G-protein-coupled receptors in the human genome form five main families. Phylogenetic analysis, paralogon groups, and fingerprints. Mol Pharmacol 63: 1256-1272.

Hakak Y, Shrestha D, Goegel MC, Behan DP, Chalmers DT (2003). Global analysis of G-protein-coupled receptor signaling in human tissues. FEBS Lett 550: 11-17.

Harmar AJ, Hills RA, Rosser EM, Jones M, Buneman OP, Dunbar DR et al. (2009). IUPHAR-DB: the IUPHAR database of G proteincoupled receptors and ion channels. Nucleic Acids Res 37 (Database issue): D680-D685.

Insel PA, Zhang L, Murray F, Yokouchi H, Zambon AC (2011). Cyclic AMP is both a pro-apoptotic and anti-apoptotic scond messenger. Acta Physiol doi: 10.1111/j.1748-1716.2011.02273.x. [Epub ahead of print].
Kidd M, Modlin IM, Gustafsson BI, Drozdov I, Hauso O, Pfragner R (2008). Luminal regulation of normal and neoplastic human EC cell serotonin release is mediated by bile salts, amines, tastants, and olfactants. Am J Physiol Gastrointest Liver Physiol 295: G260-G272.

Malik NN (2009). Controlling the cost of innovative cancer therapeutics. Nat Rev Clin Oncol 6: 550-552.

Moore-Morris T, Varrault A, Mangoni ME, Le Digarcher A, Negre V, Dantec C et al. (2009). Identification of potential pharmacological targets by analysis of the comprehensive $\mathrm{G}$ protein-coupled receptor repertoire in the four cardiac chambers. Mol Pharmacol 75 : $1108-1116$.

Neuhaus EM, Zhang W, Gelis L, Deng Y, Noldus J, Hatt H (2009). Activation of an olfactory receptor inhibits proliferation of prostate cancer cells. J Biol Chem 284: 16218-16225.

Oh DY, Talukdar S, Bae EJ, Imamura T, Morinaga H, Fan W et al. (2010). GPR120 is an omega-3 fatty acid receptor mediating potent anti-inflammatory and insulin-sensitizing effects. Cell 142: 687-698.

Pluznick JL, Zou DJ, Zhang X, Yan Q, Rodriguez-Gil DJ, Eisner C et al. (2009). Functional expression of the olfactory signaling system in the kidney. Proc Natl Acad Sci USA 106: 2059-2064.

Rajagopal S, Rajagopal K, Lefkowitz RJ (2010). Teaching old receptors new tricks: biasing seven-transmembrane receptors. Nat Rev Drug Discov 9: 373-386.

Regard JB, Sato IT, Coughlin SR (2008). Anatomical profiling of G protein-coupled receptor expression. Cell 135: 561-571.

Reiners J, Schmidt M, Packer J, Unger L, Wernet W (2007). A polymorphism linked to bipolar affective disorder does not alter the CRE activity of constitutively activated trace amine receptor 4 . Mol Psychiatry 12: 900-902.

Shah AS, Ben-Shahar Y, Moninger TO, Kline JN, Welsh MJ (2009). Motile cilia of human airway epithelia are chemosensory. Science 325: 1131-1134.

Sharman JL, Mpamhanga CP, Spedding M, Germain P, Staels B, Dacquet C et al. (2011). IUPHAR-DB: new receptors and tools for easy searching and visualization of pharmacological data. Nucleic Acids Res 39 (Database issue): D534-D538.

Snead AN, Insel PA (EB 2010) Identification and validation of new protein target(s) for the regulation of cardiac fibrosis. FASEB J (Meeting Abstract Supplement) 24: 769.12.

Swaney JS, Roth DM, Olson ER, Naugle JE, Meszaros JG, Insel PA (2005). Inhibition of cardiac myofibroblast formation and collagen synthesis by activation and overexpression of adenylyl cyclase. Proc Natl Acad Sci USA 102: 437-442.

Vassilatis DK, Hohmann JG, Zeng H, Li F, Ranchalis JE, Mortrud MT et al. (2003). The G protein-coupled receptor repertoires of human and mouse. Proc Natl Acad Sci USA 100: $4903-4908$.

Wellendorph P, Johansen LD, Brauner-Osborne H (2009). Molecular pharmacology of promiscuous seven transmembrane receptors sensing organic nutrients. Mol Pharmacol 76: 453-465.

Yokoyama U, Patel HH, Lai NC, Aroonsakool N, Roth DM, Insel PA (2008). The cyclic AMP effector Epac integrates pro- and anti-fibrotic signals. Proc Natl Acad Sci USA 105: 6386-6391. 